\title{
Neutral pion form factor measurement by the NA62 experiment
}

\author{
Monica Pepe ${ }^{1,2, a}$ \\ ${ }^{1}$ INFN Sezione di Perugia \\ ${ }^{2}$ On behalf of the $N A 62-R_{K}$ and NA48/2 Collaborations
}

\begin{abstract}
The NA62 experiment at CERN collected a large sample of charged kaon decays with a highly efficient trigger for decays into electrons in 2007. A measurement of the electromagnetic transition form factor slope of the neutral pion in the time-like region from about one million fully reconstructed $\pi^{0}$ Dalitz decays is presented.

The limits on dark photon production from a sample of about $1.7 \times 10^{7} \pi^{0}$ Dalitz decays collected in 2003-2004 by the earlier kaon experiment at CERN NA48/2 are also reported.
\end{abstract}

\section{Introduction}

High intensity kaon experiments provide great opportunities for precision $\pi^{0}$ decay measurements since kaons are a source of tagged neutral pion decays. The NA48/2 experiment at CERN SPS took data in 2003-2004 using simultaneous $K^{+} / K^{-}$beams, collecting about $2 \times 10^{11} K^{ \pm}$decays in the fiducial decay volume. In 2007 a 10 times smaller $K^{ \pm}$decay sample was collected by the NA62 experiment exploiting an improved set-up of the NA48/2 detector with lower intensity beams and minimum bias trigger conditions.

This paper reports neutral pion physics results from both experiments. The preliminary measurement of the $\pi^{0}$ electromagnetic transition form factor (TFF) slope parameter is based on a sample of $1.05 \times 10^{6} \pi^{0}$ Dalitz $\left(\pi_{D}^{0}\right)$ decays collected by NA62. Using a sample of $\sim 1.7 \times 10^{7}$ neutral pions tagged in $K^{ \pm} \rightarrow \pi^{ \pm} \pi_{D}^{0}$ and $K^{ \pm} \rightarrow \mu^{ \pm} \pi_{D}^{0} v$ decays, NA48/2 performed a search for the dark photon $A^{\prime}$ through the decay chain $\pi^{0} \rightarrow \gamma A^{\prime}, A^{\prime} \rightarrow e^{+} e^{-}$.

\section{Beam and detector}

The beam line of the NA48/2 experiment [1] was designed to provide simultaneous $K^{+} / K^{-}$beams: positive and negative hadron beams were produced in the same beryllium target by impinging 400 $\mathrm{GeV} / \mathrm{c}$ protons from the CERN SPS accelerator. Charged particles with momenta of $(60 \pm 3) \mathrm{GeV} / \mathrm{c}$ were selected by an achromatic system of four dipole magnets splitting the two beams in the vertical plane and recombining them on a common axis. Kaon decays were collected in a $114 \mathrm{~m}$ long fiducial decay region contained in a cylindrical vacuum tank. This line was also used by NA62 in 2007 at lower beam intensity with $K^{+}$and $K^{-}$beams alternatively produced with a momentum range $(74 \pm 1.4)$ $\mathrm{GeV} / \mathrm{c}$. The beams were mostly composed of $\pi^{ \pm}$, with a $K^{ \pm}$fraction of approximately $6 \%$.

\footnotetext{
ae-mail: monica.pepe@pg.infn.it
} 
Charged decay product momenta were measured by a magnetic spectrometer consisting of two sets of drift chambers and a central dipole magnet, providing a momentum resolution $\sigma(p) / p=$ $(1.02 \oplus 0.044 \cdot p) \%$ in 2003-2004 and $\sigma(p) / p=(0.48 \oplus 0.009 \cdot p) \%$ in $2007(p$ in $\mathrm{GeV} / \mathrm{c})$. A hodoscope consisting of two planes of plastic scintillators segmented into horizontal and vertical strips provided a fast trigger for charged particles with very good $(\sim 150 \mathrm{ps})$ time resolution. A liquid Krypton calorimeter ( $\mathrm{LKr}$ ), $27 X_{0}$ deep, was used to measure electromagnetic energy deposition of photons and electrons with a resolution $\sigma_{E} / E=(3.2 \sqrt{E} \oplus 9 \% / E \oplus 0.42) \%(E$ in $\mathrm{GeV})$. The detector was completed by a hadron calorimeter followed by a muon veto system. A detailed description of the experimental apparatus can be found in Ref.[2].

\section{Measurement of the $\pi^{0}$ electromagnetic transition form factor slope in NA62}

Hadron-photon couplings are fundamental observables in particle physics. They can be described in terms of form factors merging effects due to the underlying structure of hadrons, hence precision measurements of Transition Form Factors (TFF) are very significant probes to investigate electromagnetic interactions and the internal structure of hadrons. The $\pi^{0}$ electromagnetic TFF also enters the prediction of significant observable quantities, such as the rate of the rare decay $\pi^{0} \rightarrow e^{+} e^{-}$ and the hadronic light-by-light scattering contribution to the magnetic moment $(g-2)_{\mu}$ of the muon [3]. Improved measurements have been recently made available from studies of the radiative and Dalitz meson decays as well as from meson production in photon-photon processes: precise model independent measurements of the $\pi^{0}$ TFF slope parameter are essential to set new stricter constraints on theoretical models.

Pions are the lighest mesons, well suited to probe low energy hadron dynamics and copiously produced in kaon decays, therefore kaon experiments are ideal environments for precision studies of the pion properties: since neutral pions are produced in four of the six main decay modes of the charged kaons, the NA62 experiment can also be considered as a $\pi^{0}$ factory.

\subsection{Measurement principle}

The $\pi^{0}$ Dalitz decay $\pi_{D}^{0} \rightarrow e^{+} e^{-} \gamma$ with $\mathrm{BR}=(1.174 \pm 0.035) \%$ [4] is the second most frequent $\pi^{0}$ decay channel occurring when one of the photons has an off-shell mass above 2 electron masses. The measurement of the $\pi^{0}$ TFF slope parameter is performed in NA62 from the study of the $K^{ \pm} \rightarrow \pi^{ \pm} \pi^{0}$ $\left(K_{2 \pi}\right)$ decay followed by the $\pi_{D}^{0}$ decay. The kinematics of the $\pi_{D}^{0}$ decay can be expressed in terms of the $x$ and $y$ Dalitz variables:

$$
x=\left(\frac{M_{e e}}{m_{\pi^{0}}}\right)^{2}=\frac{\left(p_{e^{+}}+p_{e^{-}}\right)^{2}}{m_{\pi^{0}}^{2}}, y=\frac{2 p_{\pi^{0}}\left(p_{e^{+}}-p_{e^{-}}\right)}{m_{\pi^{0}}^{2}(1-x)}
$$

where the variable kinematic limits are $r^{2} \leq x \leq 1$ and $-\beta \leq y \leq \beta$, with $r=2 m_{e} / m_{\pi_{0}}$ and $\beta=$ $\left(1-r^{2} / x\right)^{1 / 2}$.

The Leading Order (LO) differential $\pi_{D}^{0}$ decay width is

$$
\frac{d^{2} \Gamma\left(\pi_{D}^{0}\right)}{d x d y}=\frac{\alpha}{4 \pi} \Gamma\left(\pi_{2 \gamma}^{0}\right) \frac{(1-x)^{3}}{x}\left(1+y^{2}+\frac{r^{2}}{x}\right)(1+\delta(x, y))|\mathcal{F}(x)|^{2}
$$

where $\Gamma\left(\pi_{2 \gamma}^{0}\right)$ is the $\pi^{0} \rightarrow \gamma \gamma$ decay width, $\delta(x, y)$ are the radiative corrections to the process and $\mathcal{F}(x)$ is the $\pi^{0}$ electromagnetic TFF describing the photon-pion coupling. Since small variations of $\mathcal{F}(x)$ are 
expected in the allowed kinematic region, it can be parametrized by a linear expression as a function of $x, \mathcal{F}(x)=(1+a x)$, where $a$ is the TFF slope parameter.

In the Vector Meson Dominance (VMD) approach $[5,6] \mathcal{F}(x)$ is dominated by the $\rho$ and $\omega$ mesons with a predicted value $a \approx m_{\pi^{0}}^{2}\left(m_{\rho}^{-2}+m_{\omega}^{-2}\right) / 2 \approx 0.03$, which is in agreement with further theoretical studies [7-10]. Radiative corrections play a crucial role in the $\pi^{0}$ TFF measurment since their effect on the differential decay rate is comparable to the TFF one: the first study of radiative corrections in the soft-photon approximation [11] was extended by adding virtual photon contributions and photon bremsstrahlung [12] and recently improved in Ref. [13] including one-loop one-photon irreducible contibutions. The latter calculations were implemented in the NA62 Monte Carlo (MC) simulation.

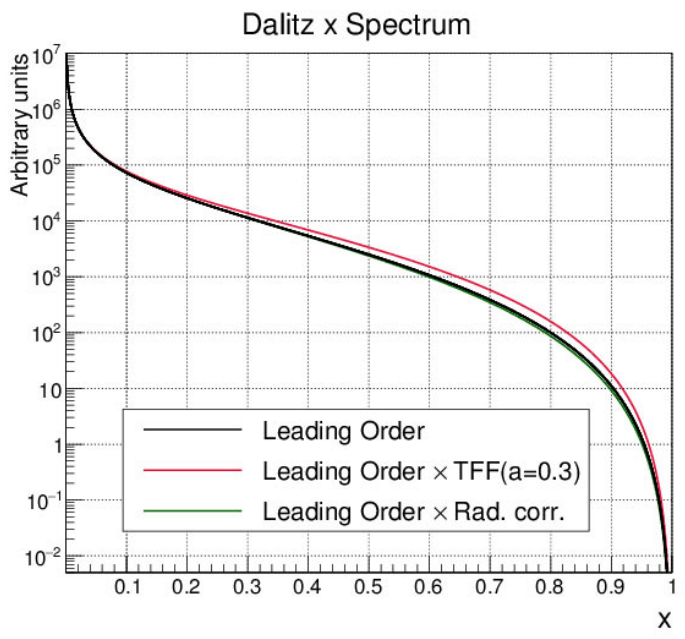

Figure 1. Predictions of the $\pi_{D}^{0}$ decay $x$ spectrum: Leading Order (black), Leading Order including $\pi_{D}^{0}$ TFF contribution with enhanced slope $a=0.3$ (red), Leading Order corrected for radiative effects (green).

Different predictions of the $\pi_{D}^{0}$ decay $x$ spectrum are compared in Fig. 1 : LO decay $x$ spectrum, LO $x$ spectrum including TFF effects with slope $a=0.3,10$ times larger than the VMD model expectation, and LO $x$ spectrum corrected for radiative effects. The three curves are very close to each other and barely distinguishable.

\subsection{The data sample}

A clean sample of $\pi^{0}$ mesons is produced in $K_{2 \pi}$ decays: the charged pion is used to tag the production of the $\pi^{0}$ and, since no undetected particles are present in the final state, background processes can be suppressed by applying stringent kinematic constraints on the reconstructed particle quantities. The data collected in 2007 by the NA62 experiment were obtained from about $2 \times 10^{10} K^{ \pm}$decays in the fiducial region, corresponding to about $5 \times 10^{9} \pi^{0}$ mesons from $K_{2 \pi}$ decays. A sample of pure $\pi_{D}^{0}$ Dalitz decays with negliglible background was collected using a spectrometer three-track vertex selection and reconstructing the photon in the $\mathrm{LKr}$ calorimeter. The final sample amounts to $1.05 \times 10^{6} \pi_{D}^{0}$ decays from $K^{ \pm} \rightarrow \pi^{ \pm} \pi_{D}^{0}\left(K_{2 \pi D}\right)$ events with a small contribution from $K^{ \pm} \rightarrow \mu^{ \pm} \pi_{D}^{0} v$ $\left(K_{\mu 3 D}\right)$ decays.

Figure 2 shows the reconstructed eer and $\pi^{ \pm} \pi^{0}$ invariant mass spectra (left and center respectively): the arrows indicate the selected region. The reconstructed spectrum of the $x$ variable 

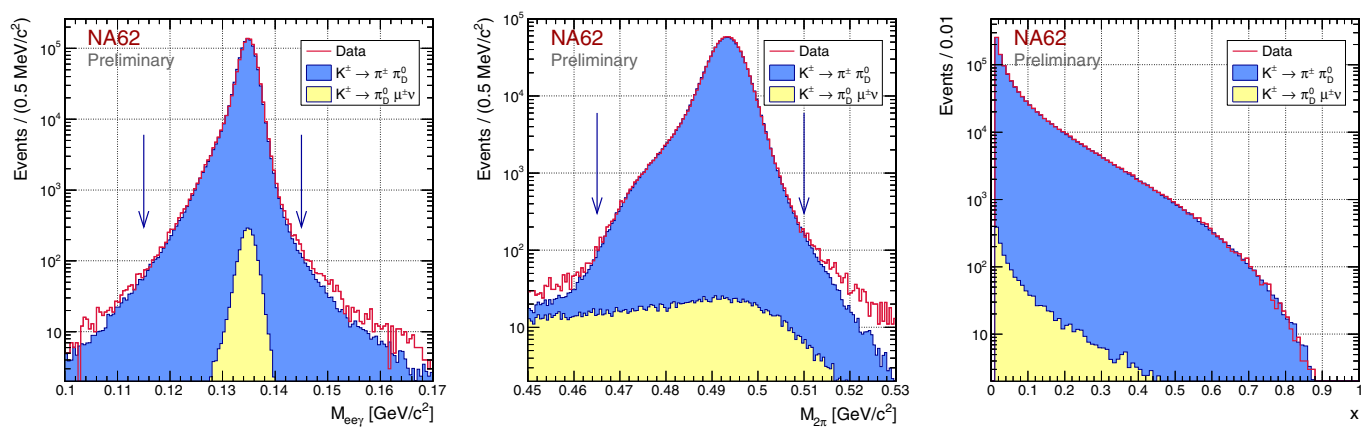

Figure 2. Reconstructed eey (left) and $\pi^{ \pm} \pi^{0}$ (center) invariant mass distributions and $x$ variable spectra (right) for data and MC components.

is shown in Fig. 2 (right) for the final sample of $\pi_{D}^{0}$ candidates compared to the MC predictions of individual contributions from $K_{2 \pi D}$ and $K_{\mu 3 D}$ decays. The acceptances evaluated with MC simulations amount to $1.81 \%$ for $K_{2 \pi D}$ and $0.02 \%$ for $K_{\mu 3 D}$ decays.

\subsection{Preliminary result}

The value of the TFF slope parameter is measured in NA62 by adjusting the MC simulation to the data in order to get the best agreement for the $\pi_{D}^{0}$ decay $x$ spectrum obtained from the normalized differential decay width (Eq. (2)) integrated over $y$.

The signal region is defined as $x>0.01$ since the event acceptance at low $x$ value is not well reproduced by the simulation: this choice does not affect the final result because the $\pi_{D}^{0} x$ spectrum is not sensitive to TFF effects for $x$ value close to zero, as shown in Fig. 1.

Table 1. Uncertainties of the $\pi^{0}$ TFF slope measurement

\begin{tabular}{ll}
\hline Source & $\delta a\left(\times 10^{2}\right)$ \\
\hline Statistical - data & 0.49 \\
Statistical - Mc & 0.20 \\
\hline Total statistics & 0.53 \\
\hline Beam momentum simulation & 0.30 \\
Spectrometer momentum scale & 0.15 \\
Spectrometer resolution & 0.05 \\
LKr non-linearity and energy scale & 0.04 \\
Particle mis-identification & 0.08 \\
Accidental background & 0.08 \\
Neglected $\pi_{D}^{0}$ sources in MC & 0.01 \\
\hline Total systematic & 0.36 \\
\hline
\end{tabular}

A $\chi^{2}$ fit to the reconstructed $x$ spectrum of data and MC simulation with different slopes is used to extract the TFF slope value using an equipopulous binning. The different hypotheses are tested by reweighing the MC events to create $x$ distributions with different slope values from the same MC sample generated using a constant TFF slope $a_{\text {sim }}=0.032$. The uncertainties on the measurement are listed in Table 1. The preliminary result is: 


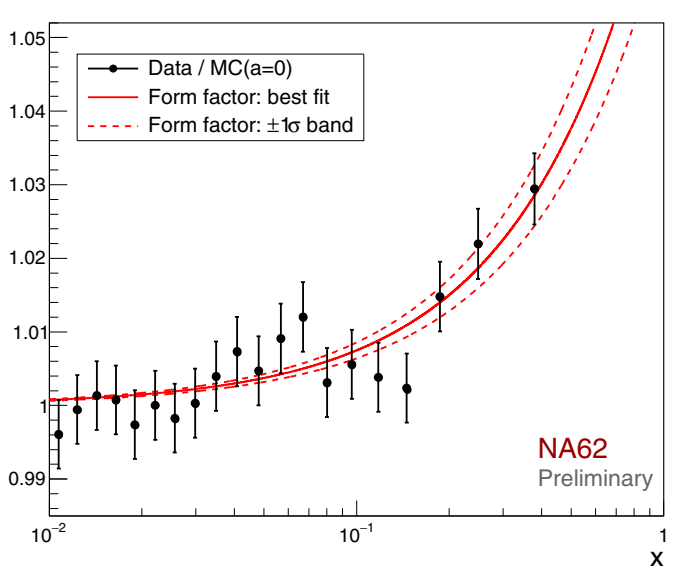

$\pi^{0}$ TFF Slope Measurements from $\pi_{\mathrm{D}}^{0}$

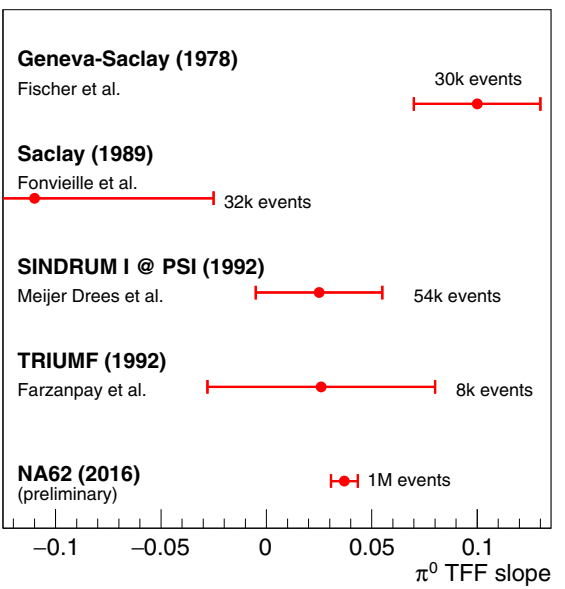

Figure 3. Left: Results of the fit to the TFF showing the data/MC ratio as a function of $x$ for a MC sample weighted to obtain $a=0$. The events are divided into 20 equipopulous bins and the marker correspond to the bin barycenter. The solid line represents $|\mathcal{F}(x)|^{2}$ with the measured slope value and the dashed lines indicate the $1 \sigma$ band.

Right: Comparison of the the NA62 preliminary measurement of the TFF slope to those of experiments exploiting the same method.

$$
a=\left(3.70 \pm 0.53_{\text {stat }} \pm 0.36_{\text {syst }}\right) \times 10^{-2}=(3.70 \pm 0.64) \times 10^{-2}
$$

The result is illustrated in Fig. 3 (left) where the effect of a positive TFF slope is clearly seen from the ratio of the reconstructed data to a MC distribution with slope $a=0$. The red solid line corresponds to a TFF function with the slope equal to the best fit central value, while dashed lines indicate the 1 $\sigma$ band. This result is the first observation (at about $5.8 \sigma$ significance) of a non-zero TFF slope in the time-like momentum transfer region and represents the more precise $\pi^{0}$ TFF slope measurement to date. The NA62 measurement can be directly compared to those of other experiments [14-17] obtained from $\pi^{0}$ Dalitz decays, as shown in Fig. 3 (right): the analysed statistics is increased by a factor of $\sim 10$ and the overall precision of the preliminary NA62 result improves the one of previous measurements.

\section{Search for dark photon in NA48/2}

The simplest hidden sector model introduces one extra U(1) gauge symmetry [18] where the interaction of a dark photon (DP) $A^{\prime}$ with the visible sector would proceed through kinetic mixing with Standard Model (SM) hypercharge. Such scenarios (with GeV-scale dark matter) provide possible explanations for the observed positron fraction excess in cosmic-rays and also offer a possible solution to the muon gyromagnetic ratio $(g-2)_{\mu}$ anomaly [19]. The DP is characterized by two a priori unknown parameters, the mass $m_{A^{\prime}}$ and the mixing parameter $\varepsilon^{2}$. It can be produced in $\pi^{0}$ decays via the chain $\pi^{0} \rightarrow \gamma A^{\prime}, A^{\prime} \rightarrow e^{+} e^{-}$with the expected branching ratio [20]:

$$
\mathcal{B}\left(\pi^{0} \rightarrow \gamma A^{\prime}\right)=2 \varepsilon^{2}\left(1-\frac{m_{A^{\prime}}^{2}}{m_{\pi^{0}}^{2}}\right)^{3} \mathcal{B}\left(\pi^{0} \rightarrow \gamma \gamma\right)
$$


which is kinematically suppressed for $m_{A^{\prime}}$ approaching the $\pi^{0}$ mass. In the DP mass range accessible in pion decays, $2 m_{e}<m_{A^{\prime}}<m_{\pi^{0}}$, the only allowed tree-level decay of the dark photon in SM fermions is $A^{\prime} \rightarrow e^{+} e^{-}$due to the high suppression of loop-induced SM decays $\left(A^{\prime} \rightarrow 3 \gamma, A^{\prime} \rightarrow v \bar{v}\right)$, hence for a DP decaying only in SM particles $\mathcal{B}\left(A^{\prime} \rightarrow e^{+} e^{-}\right) \approx 1$ and the expected total width [20] is:

$$
\Gamma_{A^{\prime}} \approx \Gamma\left(A^{\prime} \rightarrow e^{+} e^{-}\right)=\frac{1}{3} \alpha \varepsilon^{2} m_{A^{\prime}} \sqrt{1-\frac{4 m_{e}^{2}}{m_{A^{\prime}}^{2}}}\left(1+\frac{2 m_{e}^{2}}{m_{A^{\prime}}^{2}}\right)
$$

Since for $2 m_{e} \ll m_{A^{\prime}}<m_{\pi^{0}}$ the DP mean proper lifetime $\tau_{A^{\prime}}$ satisfies the relation

$$
c \tau_{A^{\prime}}=\hbar / \Gamma_{A^{\prime}} \approx 0.8 \mu m \times\left(\frac{10^{-6}}{\varepsilon^{2}}\right) \times\left(\frac{100 \mathrm{MeV} / \mathrm{c}^{2}}{m_{A^{\prime}}}\right)
$$

for sufficiently large values of $m_{A^{\prime}}$ and $\varepsilon^{2}$ the DP decay is assumed to occurr at the production point: in this case the DP decay signature is identical to that of the Dalitz decay $\pi_{D}^{0} \rightarrow e^{+} e^{-} \gamma$ (prompt decay). Therefore the $\pi_{D}^{0}$ decay represents an irreducible background and determines the measurement sensitivity.

\subsection{Event selection}

The NA48/2 experiment collected a large sample of $K^{ \pm}$decays in flight providing a sample of pure $\pi_{D}^{0}$ reconstructed through $K^{ \pm} \rightarrow \pi^{ \pm} \pi^{0}\left(K_{2 \pi}\right)$ and $K^{ \pm} \rightarrow \mu^{ \pm} \pi^{0} v\left(K_{\mu 3}\right)$ decays. The full NA48/2 data sample is used in this analysis consisting in the search for the decay chains starting from a $K_{2 \pi}$ or $K_{\mu 3}$ decay followed by the prompt decay $\pi^{0} \rightarrow \gamma A^{\prime}, A^{\prime} \rightarrow e^{+} e^{-}$. A detailed description of the event selection can be found in Ref.[21]. The selection of both decays requires three-track vertices reconstructed in the fiducial decay region and two opposite sign electrons; charged particle identification is based on the ratio of the energy deposited in the LKr calorimeter to the momentum measured by the spectrometer; a single isolated LKr energy deposition cluster defines the photon candidate. The event selection criteria for the two channels are identical up to the momentum, invariant mass and particle identification conditions applied to classify an event as a $K_{2 \pi}$ or $K_{\mu 3}$ candidate.

Table 2. Number of data events passing the $K_{2 \pi D}$ and $K_{\mu 3 D}$ selection and their acceptances computed with MC simulations. The statistical errors on the acceptances are negligible.

\begin{tabular}{lll}
\hline & $K_{2 \pi D}$ selection & $K_{\mu 3 D}$ selection \\
\hline Data Candidates & $N_{K_{2 \pi D}}=1.38 \times 10^{7}$ & $N_{K_{\mu 3 D}}=0.31 \times 10^{7}$ \\
\hline Acceptances & & \\
for $K_{2 \pi D}$ decay & $A_{\pi}\left(K_{2 \pi D}\right)=3.71 \%$ & $A_{\mu}\left(K_{2 \pi D}\right)=0.11 \%$ \\
for $K_{\mu 3 D}$ decay & $A_{\pi}\left(K_{\mu 3 D}\right)=0.03 \%$ & $A_{\mu}\left(K_{\mu 3 D}\right)=4.17 \%$ \\
for $K_{3 \pi D}$ decay & $A_{\pi}\left(K_{3 \pi D}\right)=0$ & $A_{\mu}\left(K_{3 \pi D}\right)=0.06 \%$ \\
\hline
\end{tabular}

In addition to the individual DP selections for $K_{2 \pi}$ and $K_{\mu 3}$ decays, a joint DP selection is considered for events passing either the $K_{2 \pi}$ or the $K_{\mu 3}$ criteria: the acceptance of the joint selection is the sum of the acceptances of the two mutually exclusive individual selections. The $K^{ \pm} \rightarrow \pi^{ \pm} \pi^{0} \pi^{0}\left(K_{3 \pi}\right)$ decay is considered as a background in the $K_{\mu 3}$ sample. The Dalitz decays $K_{2 \pi D}$ and $K_{\mu 3 D}$ are selected excluding the DP mass cut from the selection criteria. The number of events passing the selection criteria (taking into account cross-feeding between decay modes) are listed in Table 2 together with the relative acceptances. The number of $\pi_{D}^{0}$ candidates reconstructed with the joint Dalitz decay selection is $1.69 \times 10^{7}$. 

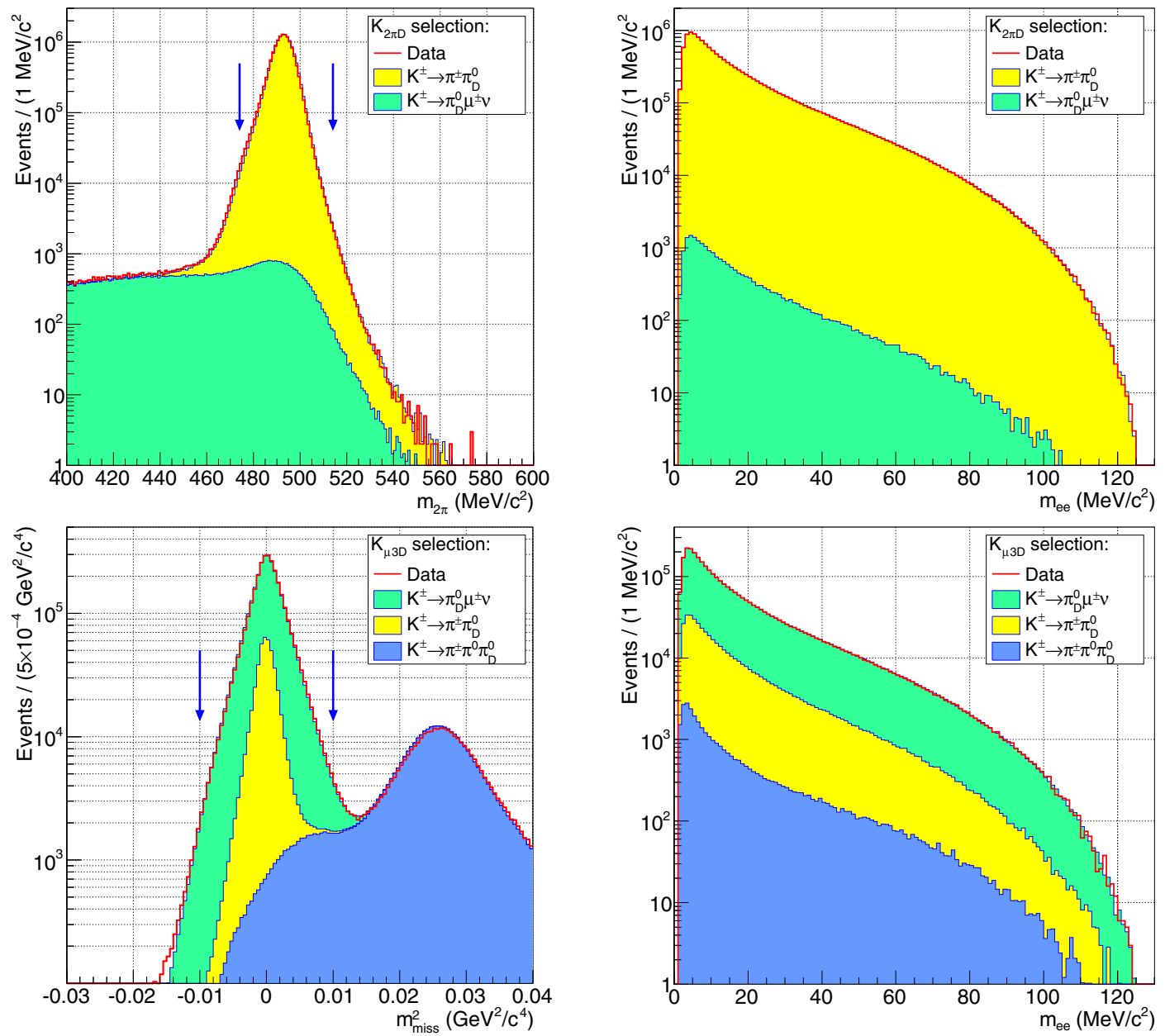

Figure 4. Invariant mass distribution of data and MC events passing the $K_{2 \pi D}$ (top row) and $K_{\mu 3 D}$ (bottom row) selections. Vertical arrows indicate the signal mass regions. A DP signal would appear as a spike in the $m_{e e}$ distributions shown in the right column.

Figure 4 shows the reconstructed invariant mass spectra for data and MC events passing the Dalitz decay selection: MC samples [21] are normalized to the data using the estimated number $N_{K}$ of total $K^{ \pm}$decays in the fiducial decay region $\left(N_{K}=(1.57 \pm 0.05) \times 10^{11}\right)$.

\subsection{Search for dark photon signal}

A scan for a DP signal is performed in the mass range $9 \mathrm{MeV} / \mathrm{c}^{2} \leq m_{A^{\prime}}<120 \mathrm{MeV} / \mathrm{c}^{2}$, where the lower boundary is determined by the limited accuracy of the $\pi_{D}^{0}$ background simulation at low $m_{e e}$. For DP masses approaching the upper limit of the mass range the sensitivity to the DP mixing parameter $\varepsilon^{2}$ is not competitive with the existing limits due to the kinematic suppression of the $\pi^{0} \rightarrow \gamma A^{\prime}$ decay. For each of the 404 considered DP masses, the number of observed data event $N_{o b s}$ passing the joint 
DP selection is compared to the estimated number of background events $N_{\text {exp }}$ evaluated from MC simulations. The local statistical significance of the DP signal for each mass hypothesis, shown in Fig. 5(Left), never exceeds 3 $\sigma$, therefore no DP signal is observed. Upper limits (UL) at 90\% CL on the number of DP candidates $N_{D P}$ for each mass value are computed from $N_{o b s}, N_{\text {exp }}$ and $\delta N_{\text {exp }}$ using the frequentist Rolke-Lopez method [22], while the upper limits at 90\% CL on the branching fraction $\mathcal{B}\left(\pi^{0} \rightarrow \gamma A^{\prime}\right)$, shown in Fig. 5(Right), are computed under the assumption that $\mathcal{B}\left(A^{\prime} \rightarrow e^{+} e^{-}\right)=1$ (which is a good approximation for $m_{A^{\prime}}<2 m_{\mu}$ if $A^{\prime}$ decays to SM fermions only). Details about the UL computation can be found in Ref. [21].
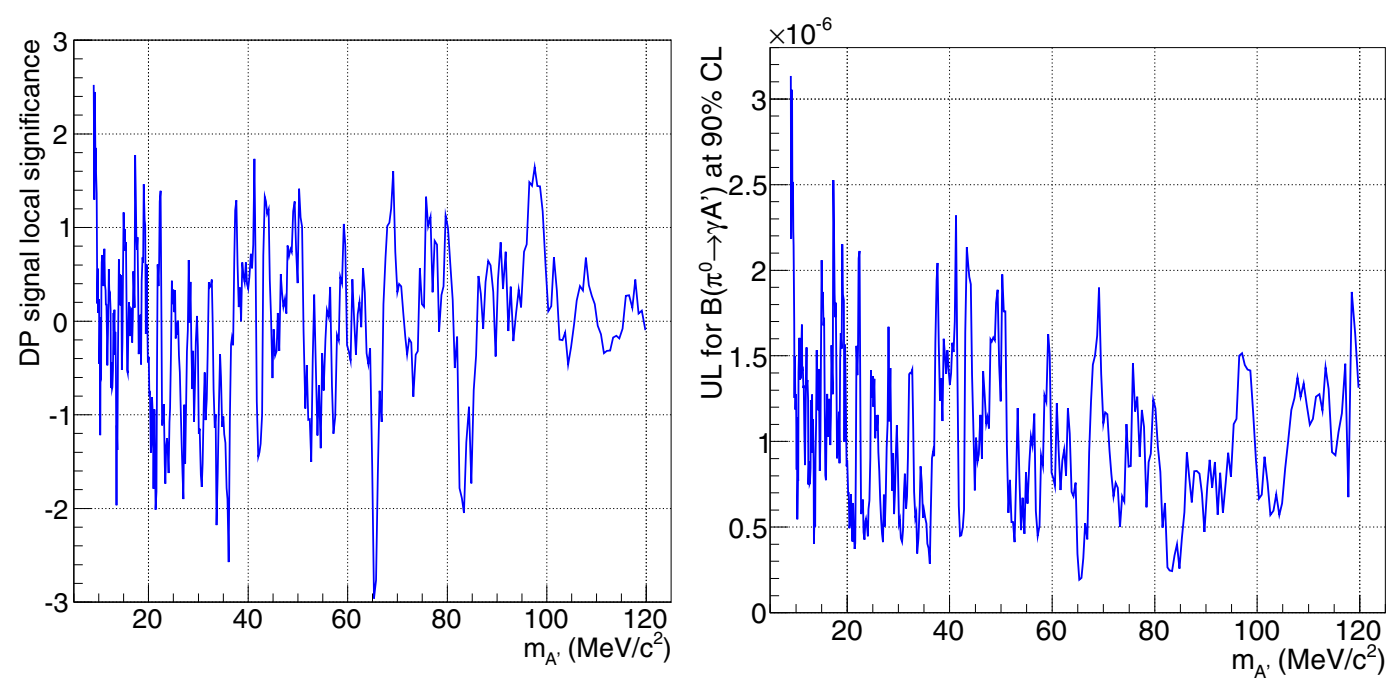

Figure 5. Left: Estimated local significance of the DP signal for each DP mass value $m_{A^{\prime}}$ : neighbouring values are strongly correlated since the mass step of the scan is about 6 times smaller than the signal window width. Right: UL at $90 \% \mathrm{CL}$ on $\mathcal{B}\left(\pi^{0} \rightarrow \gamma A^{\prime}\right)$ for each $m_{A^{\prime}}$ value.

The upper limits at $90 \% \mathrm{CL}$ on the mixing parameter $\varepsilon^{2}$ for each DP mass value are calculated from the $\mathcal{B}\left(\pi^{0} \rightarrow \gamma A^{\prime}\right)$ upper limits using Eq.(3). They are compared in Fig. 6 to other published exclusion limits [23-29]. Also shown in the figure are the band in the $\left(m_{A^{\prime}}, \varepsilon^{2}\right)$ plane where the discrepancy between the measured and calculated muon $(g-2)_{\mu}$ values falls into the $\pm 2 \sigma$ range and the region excluded by the electron $(g-2)_{e}$ measurement [19, 30,31].

This result improves the existing UL on the mixing parameter $\varepsilon^{2}$ in the $m_{A^{\prime}}$ range $9-70 \mathrm{MeV} / \mathrm{c}^{2}$. In combination with other experimental limits and under the assumption that the DP couples to quarks and mainly decays into SM fermions, this result rules out the DP as possible explanation for the muon $(g-2)_{\mu}$ anomaly.

The sensitivity on the prompt $A^{\prime}$ search is limited by the irreducible $\pi_{D}^{0}$ background. Furthermore, since the achievable UL on $\varepsilon^{2}$ scales as the inverse of the square root of the integrated beam flux, only modest improvements using the presented technique are expected with future larger $K^{ \pm}$samples.

\section{Conclusions}

The NA62 experiment has performed a preliminary measurement of the $\pi^{0}$ electromagnetic TFF in the time-like region of momentum transfer from a sample of about one million fully reconstructed $\pi_{D}^{0}$ 


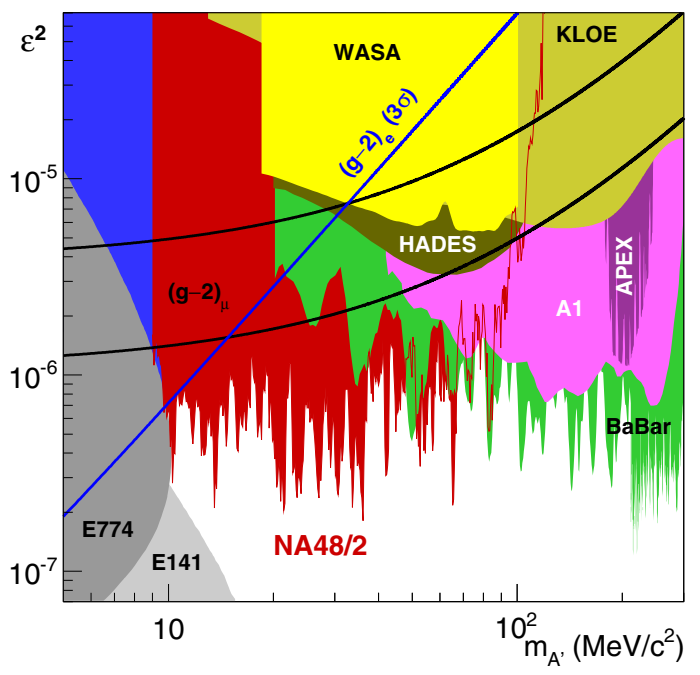

Figure 6. Upper limits at $90 \% \mathrm{CL}$ on the mixing parameter $\varepsilon^{2}$ versus the DP mass $m_{A^{\prime}}$ : the NA48/2 result is compared to other published exclusion limits.

decays collected in 2007 . The preliminary result $a=(3.70 \pm 0.64) \times 10^{-2}$, the most precise to date, is compatible with theoretical expectations and consistent with the previous measurements.

A search for dark photon $A^{\prime}$ produced in the $\pi^{0} \rightarrow \gamma A^{\prime}$ decay followed by the prompt $A^{\prime} \rightarrow e^{+} e^{-}$ decay has been performed by the NA48/2 experiment from a sample of about $1.7 \times 10^{7}$ reconstructed $\pi_{D}^{0}$ candidates, using data collected in 2003-2004. No DP signal is observed: the final NA48/2 result [21] gives the most stringent upper limits on the mixing parameter $\varepsilon^{2}$ in the $A^{\prime}$ mass range $9-70 \mathrm{MeV} / \mathrm{c}^{2}$.

\section{References}

[1] J.R. Batley et al., Eur. Phys. J. C52 875 (2007)

[2] V. Fanti et al., Nucl.Instrum.Meth. A574 433 (2007)

[3] A. Nyffeler Phys. Rev.D94 053006 (2016)

[4] C. Patrignani et al (Particle Data Group), Chin. Phys.C40 100001 (2016)

[5] M. Gell-Mann and F. Zachariasen Phys. Rev. 124953 (1961)

[6] P. Lichard Phys. Rev. D83 037503 (2011)

[7] K. Kampf et al. 2006 Eur. Phys. J. C46 191 (2006)

[8] P. Masjuan, Phys. Rev.D86 094021 (2012)

[9] M. Hoferichter et al., Eur. Phys. J. C74 3180 (2014)

[10] T. Husek and S. Leupold Eur. Phys. J. C75 586 (2015)

[11] B.E. Lautrup and J.Smith Phys. Rev. D3 1122 (1971)

[12] K.O. Mikaelian and J.Smith Phys. Rev. D5 1763 (1972)

[13] T. Husek et al., Phys. Rev. D92 054027 (2015)

[14] J. Fisher et al., Phys. Lett. B73 359 (1978)

[15] H. Fonvieille et al., Phys. Lett. B233 65 (1989) 
[16] R. Meijer Drees et al., Phys. Rev.D45 1439 (1992)

[17] H. Farzanpay et al., Phys. Lett. B278 413 (1992)

[18] B. Holdom, Phys. Lett. B166 196 (1986)

[19] M. Pospelov, Phys. Rev. D80 095002 (2009)

[20] B.Batell et al., Phys. Rev. D80 095024 (2009)

[21] J.R. Batley et al., Phys. Lett. B746 178 (2015)

[22] W.A. Rolke and A.M. Lopez, Nucl.Instrum.Meth. A458 745 (2001)

[23] S. Andreas et 1., Phys. Rev. D86 095019 (2012)

[24] D. Babusci et al., Phys. Lett. B720 111 (2013)

[25] P. Adlarson et al., Phys. Lett. B726 187 (2013)

[26] G. Agakishev et al., Phys. Lett. B731 265 (2014)

[27] H. Merkel et al., Phys. Rev. Lett 112221802 (2014)

[28] S. Abrahamyan et al., Phys. Rev. Lett 107191804 (2011)

[29] J.P. Lees et al., Phys. Rev. Lett 113201801 (2014)

[30] M. Endo et 1., Phys. Rev. D86 095029 (2012)

[31] H. Davoudiasl et 1., Phys. Rev. D89 095006 (2014) 